\title{
CONFIDENCE AND ACCURACY IN DETERMINATION OF THE CRITICAL STATE FRICTION ANGLE
}

\author{
Mohsen GhafGhazi ${ }^{\mathrm{i})}$ and Dawn Shuttle ${ }^{\mathrm{ii})}$
}

\begin{abstract}
The critical state friction angle, $\phi_{c}$, is a parameter of interest in many geotechnical analyses dealing with cohesionless soils. However, despite the triaxial test being widely used to estimate this parameter, the accuracy achievable from a limited number of triaxial tests is unknown. Accuracy is of particular interest in industry, where typically only a few soil tests are possible due to budget limitations. Statistical analysis of an extensive high quality triaxial testing program from the literature is used here to obtain guidance on the number, density range, and pressure range of triaxial tests required to reach a specified level of confidence in the critical state friction angle. The adequacy of the estimating methodology is then tested against a smaller and independent set of triaxial tests on the same sand, performed in a commercial testing laboratory. The results suggest about six drained triaxial tests are needed for routine engineering where a precision of $\pm 1.0^{\circ}$ may suffice. However, for research or high precision work, more than twenty drained triaxial tests may be needed to determine $\phi_{\mathrm{c}}$ to an accuracy of $\pm 0.5^{\circ}$ at $90 \%$ confidence. In all cases, tested samples should be uniformly distributed across the range of the soil's relative density.
\end{abstract}

Key words: cohesionless soils, critical state, friction angle, sand, strength, triaxial testing (IGC: D6/E13)

\section{INTRODUCTION}

Geotechnical predictions are sensitive to the shear strength parameters and so it is important for geotechnical engineers to have access to good parameter estimates. One widely used shear strength parameter is the critical state (or constant volume) friction angle, $\phi_{c}$, a soil property that varies over a surprisingly wide range depending on soil mineralogy and particle angularity, amongst other factors. Critical state soil mechanics is based on the idea that soils sheared to very high values of strain will eventually reach a constant void ratio, $e_{\mathrm{c}}$, termed the critical void ratio, and constant friction angle, $\phi_{c}$, termed the critical friction angle. Well established in soil mechanics since the late 1950s, critical state soil mechanics provides a basis for understanding the soil behaviour, with many soil constitutive models based on this concept. Equally, the simplest engineering problems dealing with shear strength of soils require an estimate of the critical state friction angle, even if a critical state framework is not explicitly adopted, as $\phi_{c}$ is central to stress dilatancy (which is the micromechanical process governing soil behaviour). Despite the importance of $\phi_{c}$, the literature contains little guidance on how many laboratory tests are required to determine $\phi_{\mathrm{c}}$ within the desired accuracy. This is particularly problematic in engineering practice, where typically only a limited number of soil tests are possible due to budget limitations. This situation is exacerbated by the lack of a consensus on the most accurate method of obtaining $\phi_{\mathrm{c}}$ from standard laboratory tests.

This work uses an extensive triaxial testing program from the literature to determine $\phi_{\mathrm{c}}$ using Bishop's method (1971), this being a standard method for determining $\phi_{c}$. The database chosen is unusual in the sense that it comprises a large number of tests on a single material. By performing a statistical analysis of the database, guidance is obtained on the number, density range and pressure range of triaxial tests required to reach a specified level of accuracy and confidence in $\phi_{\mathrm{c}}$. Finally, the performance of the proposed methodology for estimating the accuracy of $\phi_{\mathrm{c}}$ determination is tested against a smaller independent set of triaxial tests on the same sand, performed in a commercial testing laboratory.

\section{TRIAXIAL DATABASE}

The material used for this study was Erksak Sand, a sand used in several offshore construction projects in the Beaufort Sea (Jefferies et al., 1985). Erksak Sand is a medium $\left(D_{50}=0.34 \mathrm{~mm}\right)$, uniform $\left(U_{\mathrm{c}}=1.8\right)$ mainly quartz sand with sub-rounded particles. Testing of this sand was independently reported by Vaid and Sasitharan (1992) and Been et al. (1991).

i) PhD Candidate, Department of Civil Engineering, University of British Columbia, Canada (mohsen@civil.ubc.ca or mohsenghaf@ yahoo.com).

ii) Consultant, Canada (formerly Associate Professor, University of British Columbia).

The manuscript for this paper was received for review on April 18, 2008; approved on March $26,2009$.

Written discussions on this paper should be submitted before January 1, 2010 to the Japanese Geotechnical Society, 4-38-2, Sengoku, Bunkyoku, Tokyo 112-0011, Japan. Upon request the closing date may be extended one month. 
The majority of this work focuses on 34 drained triaxial compression tests performed in the University of British Columbia's (UBC) soils laboratory, as reported by Vaid and Sasitharan (1992). Samples were prepared using the water pluviation technique at three different relative densities; $26 \%$ (13 tests), 56\% (13 tests) and 70\% (8 tests) representing loose, medium dense and dense conditions. A smaller set of drained triaxial data are reported by Been et al. (1991), comprising 9 triaxial compression tests performed in Golder Associates Ltd. Calgary commercial testing laboratory. The Been et al. tests were also prepared using water pluviation and had relative densities ranging between $20 \%$ and $74 \%$ with five loose, two medium and two dense tests. The terms loose, medium dense and dense refer to initial relative density values of 15-35 $\%, 35-65 \%$, and $65-85 \%$ respectively. It should be mentioned that even the loosest samples tested showed "dense" sand behaviour; i.e., having a clear peak strength and dilating towards the critical state. This lack of pressure dependence on soil behaviour is a pitfall of using $D_{\mathrm{r}}$ as the primary density index. However, despite such limitations $D_{\mathrm{r}}$ is used as the density index in this work due to its simplicity, and more importantly its widespread adoption in engineering practice.

\section{METHODOLOGY USED FOR $\phi_{\mathrm{c}}$ DETERMINATION}

The critical friction angle $\phi_{\mathrm{c}}$ appears to be constant for a particular soil under triaxial compression conditions (e.g., Rowe, 1962; Negussey et al., 1988). However, there is no consensus on the most accurate method of determining its value. Ghafghazi and Shuttle (2006) discuss four different methods of determining $\phi_{\mathrm{c}}$ from triaxial compression tests. The first method, termed End of Test, assumes the measured friction angle at the end of the triaxial test is equal to $\phi_{\mathrm{c}}$. End of Test is simple, but most samples do not reach the critical state within the strain limits of the triaxial test and post-peak localisation could also render the measurements unreliable. The second method, Maximum Contraction, incorrectly assumes that the friction angle at maximum contraction is equivalent to $\phi_{\mathrm{c}}$ and so provides poor $\phi_{\mathrm{c}}$ values. The third method, Stress-Dilatancy, involves extrapolating a plot of the post-peak stress ratio versus dilatancy data to zero. This provides good predictions in the absence of localisation immediately post peak, however it is difficult to automate and involves user interpretation. The final method considered was Bishop's Method (1971), which has the advantage of yielding theoretically correct answers while being easily automated for any dataset of triaxial compression tests. This study uses Bishop's method.

Bishop's method of obtaining critical state friction angle is based on the idea that, for constant mean stress, the peak friction angle of a soil increases with increasing density, and that a purely contractive soil will reach peak strength at critical state. Bishop used the parameters $\phi$ and $d \varepsilon_{\mathrm{v}} / d \varepsilon_{1}$. However, the alternative identity $\eta$ and $D$ are preferred because these variables are, theoretically, linearly related (Schofield and Wroth, 1968; Nova, 1982).
The dilatancy, $D$, is defined as the ratio of rate of volume change to the rate of change in shear strain invariant. In mathematical form:

$$
D=\frac{d \varepsilon_{\mathrm{v}}}{d \varepsilon_{\mathrm{q}}}
$$

where $\varepsilon_{\mathrm{v}}\left[=\varepsilon_{1}+2 \varepsilon_{3}\right]$ and $\varepsilon_{\mathrm{q}}\left[=2 / 3 \times\left(\varepsilon_{1}-\varepsilon_{3}\right)\right]$ are the triaxial volumetric and deviatoric strain invariants respectively. The dilatancy at peak strength is negative because of the compression positive convention of soil mechanics. Mobilised stresses are represented by the stress ratio, $\eta$ :

$$
\eta=\frac{q}{p^{\prime}}
$$

where $q\left[=\sigma_{1}^{\prime}-\sigma_{3}^{\prime}\right]$ and $p^{\prime}\left[=\left(\sigma_{1}^{\prime}+2 \sigma_{3}^{\prime}\right) / 3\right]$ are the triaxial deviatoric stress and mean effective stress invariants respectively and $\sigma_{1}^{\prime}, \sigma_{2}^{\prime}$ and $\sigma_{3}^{\prime}$ are the three principal effective stresses.

For known stress conditions the friction angle is directly related to the stress ratio at the critical state and the two parameters can be applied interchangeably; for example, in triaxial compression we have

$$
\phi_{\mathrm{c}}=\arcsin \left(\frac{3 M_{\mathrm{tc}}}{6+M_{\mathrm{tc}}}\right)
$$

where $M_{\mathrm{tc}}$ is the stress ratio $\eta$ at the critical state under triaxial compression conditions.

With Bishop's method, a series of triaxial tests at differing densities are carried out. As illustrated in Fig. 1, for each test the mobilised stress ratio, $\eta$, is plotted against dilatancy, $D$, and the peak point of the plot is chosen to represent the test on a plot of $\eta_{\max }$ vs. $D_{\min } . M_{\mathrm{tc}}$ is determined by extrapolation of a linear regression through the $D_{\min }-\eta_{\max }$ for all the 34 triaxial tests, with $M_{\text {tc }}$ being the intercept at zero dilatancy, as shown in Fig. 2 . For a soil sample that reaches the critical state directly, i.e., without dilation, $\eta_{\max }$ corresponds with the critical state.

In this work it is assumed that the best fit regression line through all 34 Vaid and Sasitharan tests provides the "correct" answer. The trendline shown in Fig. 2 yields

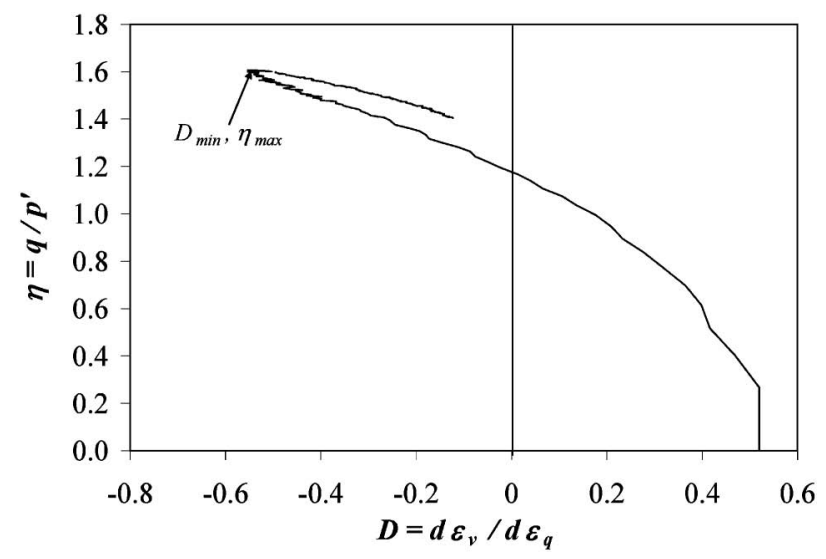

Fig. 1. Stress-Dilatancy plot for test D-667 (Been et al., 1991) on Erksak sand 


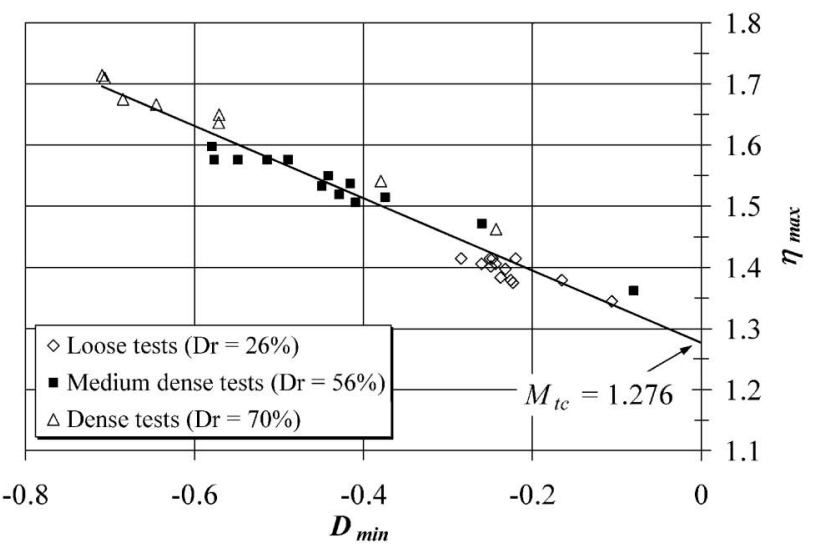

Fig. 2. $\eta_{\max }$ vs. $D_{\min }$ of 34 triaxial compression tests on Erksak sand (after Vaid and Sasitharan, 1992)

$M_{\mathrm{tc}}=1.276\left(\right.$ or $\left.\phi_{\mathrm{c}}=31.75^{\circ}\right)$ with a coefficient of determination, $R^{2}=0.955$.

\section{DETERMINATION OF $\phi_{\mathrm{c}}$ FROM LIMITED NUMBER OF TRIAXIAL TESTS}

To determine accuracy in $\phi_{\mathrm{c}}$ determination from fewer triaxial tests the simplest method would be to randomly sample the required number of tests from the database of 34 tests. However, this approach was felt to be unrealistic as most testing programs include a range of soil densities. Also, Bishop's approach is most accurate when the tests are spread over a range of $D_{\min }$; using random tests would result in some unrepresentatively poor estimates of $\phi_{c}$. Hence it was assumed that every combination of tests used to determine $\phi_{c}$ include at least one loose, one medium dense and one dense sample (i.e., one test at $D_{\mathrm{r}}=$ $26 \%, 56 \%$ and $70 \%$ respectively for the Vaid and Sasitharan dataset). Additionally, no repetition was allowed in this procedure so that no test could be sampled twice in any realisation.

Adopting this statistical methodology lead to $\phi_{\mathrm{c}}$ being calculated with $3,4,5, \ldots, 34$ tests. Typically 3 to 20 tests are presented here: 20 tests being considered an upper bound for most practical testing programs. Each combination of loose, medium dense and dense tests comprising the total number of required tests was sampled 300 times. For example, 4 tests can comprise either 1 loose, 1 medium, 2 dense or 1 loose, 2 medium, 1 dense or 2 loose, 1 medium, 1 dense tests. After each of these three possible combinations was randomly realised 300 times, $M_{\mathrm{tc}}$ was again calculated from linear regression through the realised data points. Values of $M_{\mathrm{tc}}$ were then converted to $\phi_{\mathrm{c}}$ using Eq. (3).

The precision of the $\phi_{\mathrm{c}}$ calculation, plotted at the percentage of tests falling within 0.1 degree bins, is shown in Fig. 3 for 5, 10, 15 and 24 tests. As expected, with an increasing number of tests the accuracy of the $\phi_{\mathrm{c}}$ prediction improves. However, Fig. 3 also indicates that there is a slight bias towards over-prediction of $\phi_{\mathrm{c}}$ for all numbers of tests. This bias is also evident in the determined $M_{\mathrm{tc}}$

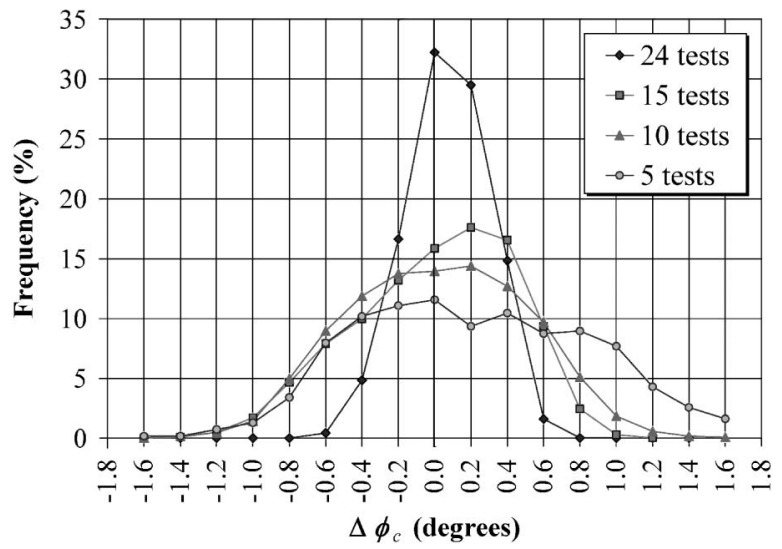

Fig. 3. Frequency of error in $\phi_{c}$ for $5,10,15$ and 24 tests

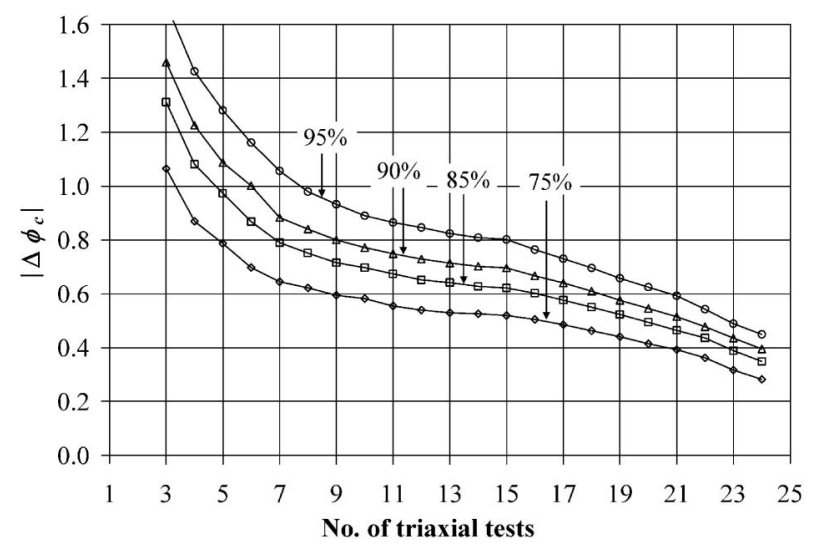

Fig. 4. Error in $\phi_{c}$ vs. number of triaxial tests at different confidence levels

data and may be related to the use of a real dataset. The magnitude of this bias is small: for 10 tests the bias in the results from quoting the average absolute error of 0.36 degrees, rather than the average over and under-prediction error of 0.39 and 0.33 degrees respectively, is only 0.03 . Hence the bias is ignored in the remainder of this work.

Figure 4 plots $\left|\Delta \phi_{\mathrm{c}}\right|$ versus number of tests for a range of confidence levels. At each confidence level the error rapidly decreases from 3 tests to around 8 tests. Thereafter a slower, almost linear, enhancement in accuracy is indicated. At the $85 \%$ confidence level this corresponds to a \pm 1.31 degree accuracy for 3 tests to better than \pm 0.75 degrees accuracy with 8 tests. The same information is provided in numerical form in Table 1 at $75 \%$, $80 \%, 85 \%, 90 \%$, and $95 \%$ confidence levels for 3 to 20 tests.

As discussed earlier, Fig. 4 is obtained using one loose, medium dense and dense test in each realisation with the remaining tests randomly distributed. From a practical perspective it is of interest to determine whether improved accuracy could be obtained by further specifying the initial densities of the samples.

Figure 5 plots the magnitude of the average and standard deviation absolute error for all possible combina- 
Table 1. Confidence level, $\left|\Delta \phi_{\mathrm{c}}\right|$, versus number of triaxial tests

\begin{tabular}{|c|c|c|c|c|c|c|c|c|c|c|c|c|c|c|c|c|c|c|c|}
\hline \multicolumn{2}{|c|}{ Number of tests } & 3 & 4 & 5 & 6 & 7 & 8 & 9 & 10 & 11 & 12 & 13 & 14 & 15 & 16 & 17 & 18 & 19 & 20 \\
\hline \multirow{5}{*}{$\begin{array}{l}\text { Confidence } \\
\text { Level (\%) }\end{array}$} & 95 & 1.67 & 1.42 & 1.28 & 1.16 & 1.06 & 0.98 & 0.93 & 0.89 & 0.86 & 0.85 & 0.82 & 0.81 & 0.80 & 0.76 & 0.73 & 0.69 & 0.66 & 0.62 \\
\hline & 90 & 1.46 & 1.22 & 1.09 & 1.00 & 0.88 & 0.84 & 0.80 & 0.77 & 0.75 & 0.73 & 0.71 & 0.70 & 0.69 & 0.67 & 0.64 & 0.61 & 0.57 & 0.54 \\
\hline & 85 & 1.31 & 1.08 & 0.97 & 0.87 & 0.79 & 0.75 & 0.71 & 0.70 & 0.67 & 0.65 & 0.64 & 0.63 & 0.62 & 0.60 & 0.58 & 0.55 & 0.52 & 0.49 \\
\hline & 80 & 1.18 & 0.98 & 0.88 & 0.77 & 0.71 & 0.69 & 0.65 & 0.63 & 0.61 & 0.59 & 0.58 & 0.57 & 0.57 & 0.55 & 0.53 & 0.50 & 0.48 & 0.45 \\
\hline & 75 & 1.06 & 0.87 & 0.79 & 0.70 & 0.64 & 0.62 & 0.59 & 0.58 & 0.55 & 0.54 & 0.52 & 0.52 & 0.52 & 0.50 & 0.48 & 0.46 & 0.44 & 0.41 \\
\hline
\end{tabular}

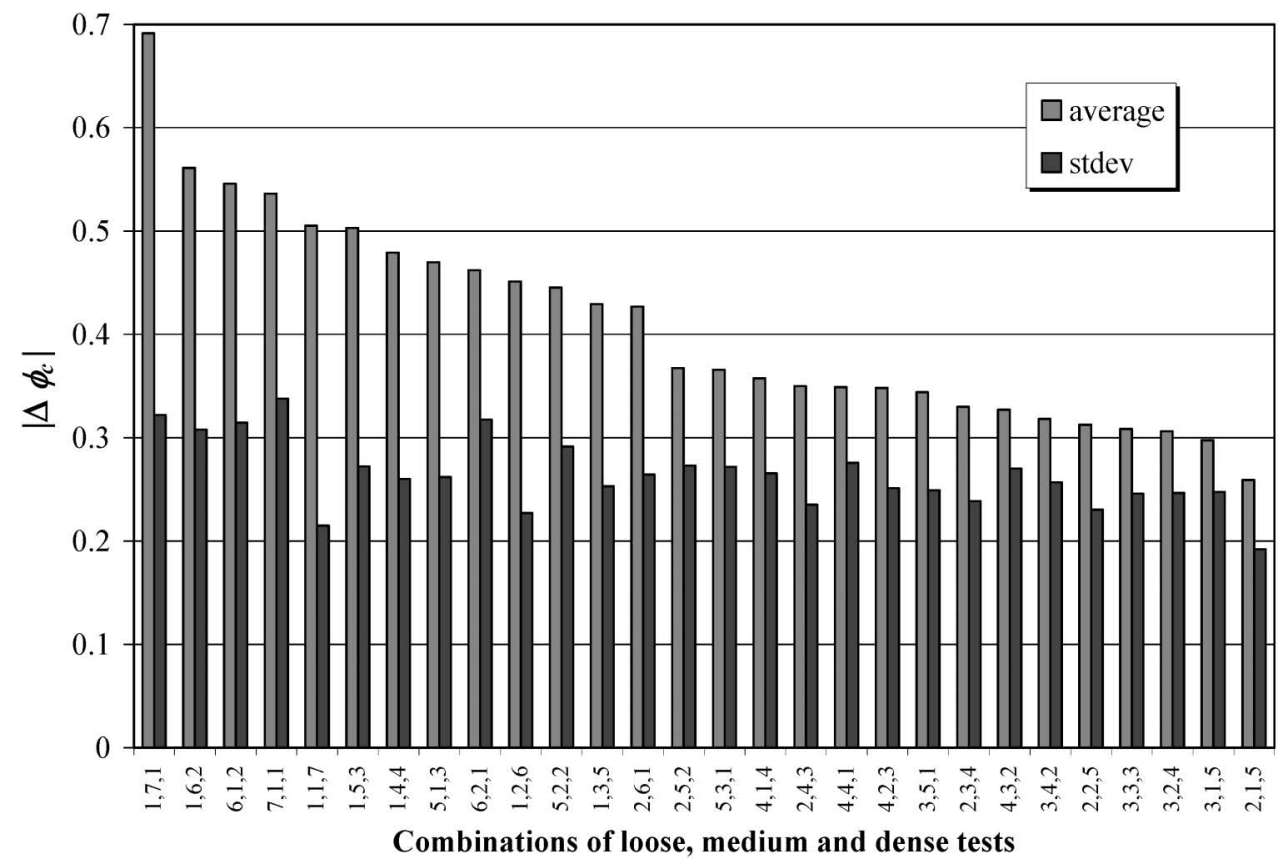

Fig. 5. Error in calculation of $\phi_{\mathrm{c}}$ obtained from 9 tests with different combinations of loose, medium and dense tests

tions of 9 tests including at least one loose, one medium and one dense test. The combinations are organised with the largest absolute error on the left hand side of the figure. The results show that of the 28 possible combinations of tests, the 10 tests with the largest error have only one from each grouping. Moreover, the three combinations with 7 tests from one density grouping are among the worst 5 groups. Conversely, the average error is approximately half where the tests are better distributed between the three groups. Hence it is advisable that the program be designed to distribute tests equally between loose, medium and dense samples.

\section{VALIDATION AGAINST INDEPENDENT TRIAXIAL DATABASE}

A high quality university testing program was used to compute the confidence levels shown in Table 1. Hence these confidence levels could be considered an upper bound on likely commercial accuracy. The general applicability of the confidence levels is tested using nine good quality commercial tests reported by Been et al. (1991). The full suite of nine tests shown in Fig. 6 indicate $M_{\mathrm{tc}}=1.254$, corresponding to $\phi_{\mathrm{c}}=31.24^{\circ}$ and suggesting an error compared to the original database of $\Delta \phi_{\mathrm{c}}=0.51^{\circ}$

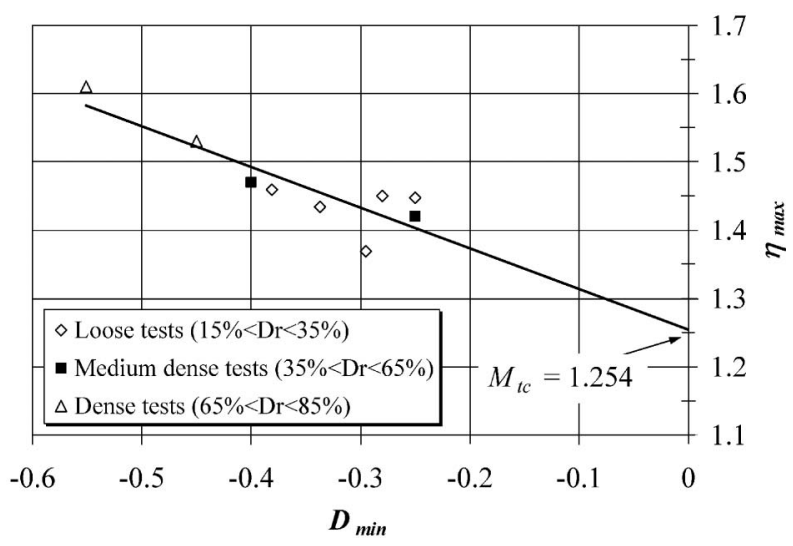

Fig. 6. $\eta_{\max }$ vs. $D_{\min }$ of 9 triaxial compression tests on Erksak sand (after Been et al., 1991)

(associated with 67\% level of confidence).

To directly compare with the predicted confidence levels it is necessary to consider possible subsets of this data. If six tests are considered, there are 70 possible combinations of tests which include at least one loose, medium dense and dense test. Therefore from Table 1 we would expect 52 of the combinations to predict $\phi_{\mathrm{c}}$ within an accuracy of $\pm 0.70^{\circ}$ at $75 \%$ confidence. The Been et al. data 
Table 2. Confidence levels for 6 tests from the independent Been et al. (1991) dataset

\begin{tabular}{l|c|c|c|c|c}
\hline Confidence Level (\%) & 75 & 80 & 85 & 90 & 95 \\
\hline Error $\left|\Delta \phi_{\mathrm{c}}\right|$ (degrees) & 1.22 & 1.29 & 1.39 & 1.96 & 2.00 \\
\hline
\end{tabular}

Table 3. Summary of initial conditions for Been et al. (1991) triaxial tests

\begin{tabular}{l|c|c|c|c|c}
\hline Test Name & Void ratio $e$ & $p^{\prime}(\mathrm{kPa})$ & $D_{\mathrm{r}}(\%)$ & $\psi$ & $D_{\min }$ \\
\hline CID G666 & 0.707 & 60 & 20.4 & -0.055 & -0.28 \\
CID G665 & 0.687 & 130 & 29.2 & -0.063 & -0.295 \\
CID G661 & 0.676 & 140 & 34.1 & -0.073 & -0.250 \\
CID G662 & 0.675 & 60 & 34.5 & -0.087 & -0.381 \\
CID G663 $^{1}$ & 0.671 & 300 & 36.3 & -0.066 & -0.337 \\
CID G761 $^{1}$ & 0.649 & 250 & 46.0 & -0.091 & -0.250 \\
CID G664 $^{1}$ & 0.630 & 300 & 54.4 & -0.107 & -0.400 \\
CID G762 $^{1}$ & 0.601 & 250 & 67.3 & -0.139 & -0.450 \\
CID G667 $^{2}$ & 0.587 & 130 & 73.5 & -0.163 & -0.551 \\
\hline
\end{tabular}

${ }^{1}$ The two tests G761 and G762 are performed on samples with overconsolidation ratio $=4$

suggest a lower accuracy of only $\pm 1.22^{\circ}$ at $75 \%$ confidence (see Table 2).

Some reasons for the lower accuracy may be observed from Fig. 6. The accuracy predicted from the Vaid and Sasitharan data is predicated on having one test from each density range. Although this criterion has been enforced for the Been et al. dataset as well, the effect of stress level (see Table 3) has resulted in one of the two medium dense tests being less dilative than all of the loose tests. Therefore the range of $D_{\min }$ between density ranges is reduced. Although this problem also exists for the large dataset, the few medium dense tests in the small dataset increase the inaccuracy. Additionally, comparison of Fig. 2 with Fig. 6 indicates that all of the "dense" tests for the Golder dataset are significantly less dilatant $\left(D_{\min }>-0.55\right)$ than the dense samples from the larger dataset $\left(D_{\min }<-0.55\right.$ for all but two samples). This problem could be reduced by maximising the range of $D_{\min }$ in Fig. 6 by ensuring that for each confining pressure at least one loose and one dense test are undertaken. This is equivalent to defining a range of state parameter, $\psi\left[=e-e_{c}\right]$, which accounts for the stress level effect on dilatancy, in addition to the void ratio accounted for in $D_{\mathrm{r}}$.

\section{SUMMARY AND CONCLUSIONS}

A statistical evaluation of a drained triaxial compression test database was performed to determine accuracy and confidence level in determining the critical state friction angle.

Critical state friction angle was obtained from a dataset comprising 34 triaxial tests using the methodology proposed by Bishop (1971); $\phi_{c}$ being obtained using linear regression. It was assumed that the correct $\phi_{\mathrm{c}}$ was obtained if all 34 tests were included in the analysis. In determining the accuracy of smaller realisations of the dataset it was assumed that any test program will include at least one loose $\left(D_{\mathrm{r}}=26 \%\right)$, one medium dense $\left(D_{\mathrm{r}}=56\right.$ $\%)$ and one dense $\left(D_{\mathrm{r}}=70 \%\right)$ sample tested under drained triaxial compression conditions.

Results were presented as error in $\phi_{\mathrm{c}}$ versus number of tests for confidence levels of $75 \%, 85 \%, 90 \%$ and $95 \%$. As the number of tests increased from 3 to 8 a large increase in accuracy was observed at all confidence levels. Hence it is recommended that any commercial testing program for evaluation of the critical state friction angle includes at least 6 tests ( 6 tests yielding an accuracy of $\pm 1.0^{\circ}$ from university quality data with $90 \%$ confidence). For academic purposes, where accuracy of $\pm 0.5^{\circ}$ with $90 \%$ confidence may be needed, more than 20 tests may be required.

Although the presented results were developed using one comprehensive academic testing program, their application to commercial data was encouraging. Although (unsurprisingly) the errors from the commercial dataset were slightly larger, the academic database provided a reasonable upper bound on likely achievable accuracy.

In conclusion, although soil type and gradation might be expected to affect sample uniformity during reconstitution and hence influence the repeatability (and hence accuracy) of the triaxial testing program, distributing the triaxial tests over a wide range of initial $D_{\mathrm{r}}$, or ideally initial $\psi$, should provide greater accuracy in $\phi_{\mathrm{c}}$ for fewer tests.

\section{ACKNOWLEDGEMENTS}

The authors wish to thank Golder Associates Ltd. for providing electronic copies of the Been et al. data. Support for this work was provided by the Natural Sciences and Engineering Research Council of Canada.

\section{REFERENCES}

1) Been, K., Jefferies, M. G. and Hachey, J. E. (1991): The critical state of sands, Géotechnique, 41(3), 365-381.

2) Bishop, A. W. (1971): Shear strength parameters for undisturbed and remoulded soil specimens, Proc. Roscoe Memorial Symposium, Cambridge, 3-58.

3) Ghafghazi, M. and Shuttle, D. A. (2006): Accurate determination of the critical state friction angle from triaxial tests, Proc. 59th Canadian Geotechnical Conference, Vancouver, 278-284.

4) Jefferies, M. G., Stewart, H. R., Thomson, R. A. A. and Rogers, B. T. (1985): Molikpaq deployment at Tarsiut P-45, Proc. ASCE Specialty Conference on Civil Engineering in the Arctic Offshore, San Francisco, CA, 1-27.

5) Negussey, D., Wijewickreme, W. K. D. and Vaid, Y. P. (1988): Constant volume friction angle of granular materials, Canadian Geotechnical Journal, 25, 50-55.

6) Nova, R. (1982): A constitutive model under monotonic and cyclic loading, Soil Mechanics-Transient and Cyclic Loads (eds. by Pande and Zienkiewicz), Wiley, 343-373.

7) Rowe, P. W. (1962): The Stress Dilatancy Relation for Static Equilibrium of an assembly of particles in Contact, Proc. Royal Society of London, Series A, 269, 500-527.

8) Schofield, A. N. and Wroth, C. P. (1968): Critical State Soil Mechanics, McGraw-Hill.

9) Vaid, Y. P. and Sasitharan, S. (1992): The strength and dilatancy of sand, Canadian Geotechnical Journal, 29, 522-526. 